UDC $615.12: 339.137$

DOI: $10.15587 / 2519-4852.2021 .239389$

\title{
SCIENTIFIC AND METHODOLOGICAL APPROACHES TO MODELING THE OPTIMAL STRATEGY FOR INCREASING THE COMPETITIVENESS OF PHARMACY CHAINS OF DIFFERENT SIZES
}

\author{
Iryna Bondarieva, Volodymyr Malyi, Olga Posilkina, Zhanna Mala, Maryna Nessonova
}

\begin{abstract}
The aim of the work is to develop scientific and methodological approaches to modelling the optimal strategy to increase the competitiveness of pharmacy chains $(P C)$, which belong to different clusters.

Materials and methods. The algorithm for determining the optimal strategy for increasing the competitiveness of PC for different clusters using the method of constructing a decision tree and cluster analysis is proposed. To solve this problem, an expert survey of more than 400 pharmacy managers, who were part of the PC of different sizes, was previously conducted. According to the results of an expert survey using hierarchical clustering methods based on the values of 13 input variables - scores of the strengths of the competitiveness of the PC, three clusters of networks were identified, each of which proposed its own algorithm for modelling the optimal strategy of competitiveness.

Results. Using modern economic and mathematical tools, the distribution of PC depending on their size into clusters for modelling the dynamics of competitiveness is substantiated. Indicators are identified, which show a significant difference between clusters, which was taken into account in the process of modelling and selection of the optimal strategy to increase the competitiveness of PC. It is established that the biggest negative impact on the strategy of increasing the competitiveness of small networks has a slow response to changes in market conditions, the biggest positive impact the availability of additional services in the networks; for medium PC the most important factors influencing the level of competitiveness are the location of pharmacies and competent management; for large PC - the use of modern automated management programs, the level of efficiency of the marketing complex and location features.

The algorithm of the generalized model of "decision tree" for a choice of optimum strategy of increase of competitiveness depending on the size of PC is constructed. It was found that the following factors are of the greatest importance: the size of the PC, the use of the discount card system, and the least - the speed of response to market changes and the stability of the financial condition.

Conclusions. The proposed generalized mathematical model of the "decision tree" allows a reasonable approach to choosing the optimal strategy to increase the competitiveness of PC depending on its size. The assessment of the importance of predictor variables for each cluster of PC allows determining the priority factors in the implementation of measures aimed at implementing the chosen strategy to increase competitiveness
\end{abstract}

Keywords: strategy, competitiveness, pharmacy chains, decision tree, clusters

How to cite:

Bondarieva, I., Malyi, V., Posilkina, O., Mala, Z., Nessonova, M. (2021). Scientific and methodological approaches to modeling the optimal strategy for increasing the competitiveness of pharmacy chains of different sizes. ScienceRise: Pharmaceutical Science, 4 (32), 59-66. doi: http://doi.org/10.15587/2519-4852.2021.239389

(C) The Author(s) 2021

This is an open access article under the Creative Commons CC BY license hydrate

\section{Introduction}

Substantiation of the strategy is the most important condition for the successful operation of each PC (pharmacy chain), as it determines the priorities of the organization for a relatively long time. Therefore, it is extremely important for management to approach the process of strategy development and implementation in a balanced manner, taking into account many internal and external factors and concentrating on the main factors. A deep understanding of the importance of this moment will allow PC to respond in a timely manner to changes in the business environment, ensure the formation and development of competitive advantages, maximize the strategic potential of the organization, and help improve medical care. Therefore, the question of defining a strat- egy to increase competitiveness for PCs, regardless of their size, is quite relevant.

Various aspects of the implementation of strategic management in the activities of pharmacies and, in particular, PC have been studied in the works of many domestic and foreign scientists. Thus, the publication [1] explores methodological approaches to the analysis and evaluation of marketing competitive advantages of PC; procedure for supporting the development of medical devices: a standard model of the product design process are presented in the publication [2]; in the publication [3] the pharmacy as an environment of pharmaceutical service is estimated. Publications [4, 5] investigate strategies and models of distribution of pharmaceutical supplies. Reconfiguration of global networks of pharmaceu- 
tical value is presented in [6]. The publication [7] developed models of flexible supply chain in the pharmaceutical industry. In [8] the methodology of research of operational management with use of quantitative modelling is resulted. Quantitative models of sustainable supply chain management have been studied in [9]; in the publication [10] presents management studies of the supply chain of pharmaceuticals. Integrated supply chain management and prospects for oligopolistic competition with the application to pharmaceuticals are presented in the publication [11]. Peculiarities of effective product range management depending on different PC sizes and the responsibility of pharmaceutical companies have been studied in $[12,13]$. Methods of structuring problems in the activities of pharmacies and the movement of analytics have been studied in publications $[14,15]$. The approach based on DEA-analysis of the competitive environment in global operating strategies is presented in the publication [16].

However, the formation of a strategy to increase the competitiveness of the PC based on determining the priority factors in the implementation of measures aimed at implementing the chosen strategy, remain out of the attention of scientists.

The aim of the work is to determine the optimal strategy to increase the competitiveness of PCs, which in terms of sales belong to different clusters.

\section{Research planning (methodology)}

In the course of the research, the priority of the strengths of competing PCs was analyzed based on a survey of more than 400 experts.

To model the dynamics of competitiveness, the division of PCs by the method of cluster analysis depending on their size into clusters (small, medium, large and mega-PC) is substantiated.

To determine the optimal strategy to increase competitiveness for small, medium, large and mega-PC, decision tree models were built. When developing mathematical models to increase the competitiveness of PC, the best results in terms of accuracy and adequacy of models were obtained using the classification tree [17].

\section{Materials and methods}

The methods of functional, logical and economicmathematical modelling, methods of mathematical statistics, questionnaires are used in the work.

To study the opinion of PC managers and employees on determining the optimal strategy to increase the competitiveness of PC, a survey was conducted according to a questionnaire developed by the authors, which included 20 questions.

Statistical methods of processing the results of the study and their analysis included the calculation of the characteristics of central tendencies, deviations from the centre and the spreads for scores of the strengths of the PC; determining the consistency of expert opinions using the Kendall concordance coefficient; methods of cluster analysis to identify homogeneous groups in the data; methods of Data Mining classification trees to determine the optimal strategies to increase competitiveness for PCs from different clusters. The number of experts was more than 400 people, i.e. it is sufficient to obtain a marginal sampling error of no more than $5.0 \%$. The reliability of the obtained results is characterized by the appropriate degree of consistency of the answers of experts in assessing the factors under study. The level of consistency of the experts' answers was determined using a concordance coefficient that was equal to 0.83 which indicates a high level of expert consensus [18].

As a result of the research, three clusters of the studied PCs were identified using hierarchical clustering methods. The linkage rule was determined by Ward's method, Euclidean distance, city-block Manhattan distance, Chebyshev's metric and percentage of disagreement were used as metrics. The quantitative composition of the clusters was determined by the K-means method, in which the initial centers of the clusters were chosen based on the principle of maximizing the distances between them. The main difference in the conclusions of experts on different clusters is due to their different assessments of the importance of the strengths of competing PCs [17].

Decision Trees models have been developed to define a strategy to increase competitiveness for small, medium, large and mega-PCs. In all clusters, the C\&RT algorithm was used to construct the decision tree, which is the most accurate in the case of binary predictor variables. As an indicator of the quality of classification in this algorithm, we used the Gini measure. To prune decision trees when developing models in clusters 1 and 2, the FACTstyle direct stopping rule with the proportion of unclassified observations not more than $1 \%$ was used. The optimal Decision Tree for cluster 3 was obtained using pruning on mis classification error with the parameters of the minimum number of observations $\mathrm{n}=0$ and the standard error rule $=0.5$. Construction and research of decision tree models were performed using the Multivariate Exploratory Techniques module of StatSoft Statistica 13. The accuracy of the developed models was evaluated in the training sample using a cross-validation procedure [17].

\section{Research results}

To model the dynamics of PC competitiveness depending on their size (small; medium; large and megaPC), more than 400 employees of 30 PCs were surveyed. Statistical characteristics of the strengths of the studied PCs are given in Tab. 1 in descending order of the values of the average ranks, by which you can set the weight of each criterion. All statistical conclusions were made with a $95 \%$ confidence level.

Since the study involved experts from the PC, which differ significantly in size, turnover, it was necessary to obtain a distribution of the entire sample by cluster based on the values of 13 input variables - assessments of competitiveness strengths to investigate differences in priorities of PC competitiveness. Because of calculations, three PC clusters were identified depending on the priorities of the factors influencing their competitiveness: small PCs form the first cluster; the second cluster - form a medium-sized PC; the third cluster most pharmacies, which belong to the large and megaPC [1]. Indicators that show a significant difference between clusters are highlighted. 
Table 1

Statistical characteristics of PC strengths

\begin{tabular}{|l|c|c|c|c|}
\hline \multicolumn{1}{|c|}{ Indicator } & $\mathrm{M} \pm \mathrm{m}$ & $\mathrm{Me}[\mathrm{UQ} ; \mathrm{LQ}]$ & $\mathrm{V}$ & $\mathrm{R}$ \\
\hline convenience of the location of the pharmacy & $3.11 \pm 0.10$ & $4.0[1.0 ; 5.0]$ & 62.63 & $\mathbf{8 . 2 3}$ \\
\hline the breadth of the range of drugs and parapharmaceuticals & $3.09 \pm 0.09$ & $4.0[2.0 ; 5.0]$ & 60.35 & $\mathbf{8 . 1 1}$ \\
\hline availability of prices for goods and services & $2.86 \pm 0.09$ & $3.0[1.0 ; 5.0]$ & 66.03 & $\mathbf{7 . 5 0}$ \\
\hline availability of a system of discounts & $2.69 \pm 0.09$ & $3.0[1.0 ; 4.0]$ & 70.25 & $\mathbf{7 . 0 8}$ \\
\hline availability of additional services & $2.70 \pm 0.09$ & $3.0[1.0 ; 5.0]$ & 69.78 & $\mathbf{7 . 0 7}$ \\
\hline the level of salaries of employees & $2.54 \pm 0.10$ & $3.0[0.0 ; 5.0]$ & 79.32 & $\mathbf{6 . 9 5}$ \\
\hline speed and quality of service & $2.55 \pm 0.10$ & $3.0[0.0 ; 4.0]$ & 76.02 & $\mathbf{6 . 8 8}$ \\
\hline microclimate in the team & $2.47 \pm 0.10$ & $3.0[0.0 ; 4.0]$ & 78.82 & $\mathbf{6 . 8 3}$ \\
\hline convenience of the schedule of work of pharmacies & $2.56 \pm 0.10$ & $3.0[1.0 ; 4.0]$ & 75.36 & $\mathbf{6 . 8 2}$ \\
\hline availability of staff training programs & $2.39 \pm 0.10$ & $3.0[0.0 ; 4.0]$ & 81.30 & $\mathbf{6 . 6 6}$ \\
\hline attractive interior of trade halls and window dressing & $2.37 \pm 0.09$ & $3.0[0.0 ; 4.0]$ & 80.04 & $\mathbf{6 . 5 5}$ \\
\hline availability of modern automated programs & $2.34 \pm 0.10$ & $2.0[0.0 ; 4.0]$ & 82.68 & $\mathbf{6 . 4 2}$ \\
\hline staff turnover level & $2.17 \pm 0.09$ & $2.0[0.0 ; 4.0]$ & 84.82 & $\mathbf{5 . 9 1}$ \\
\hline Note $: M$ is the mean value, m is the error of the mean; Me - median, $L Q-$ lower quartile, UQ- upper quartile $\cdot$ Vis the coefficient of
\end{tabular}

Note: $M$ is the mean value, $m$ is the error of the mean; $M e$ - median, $L Q$-lower quartile, $U Q-$ upper quartile; $V$ is the coefficient of variation (\%); $R$ - average rank

In the process, more than 50 possible factors influencing the dynamics of PC competitiveness were studied, which characterize the features of its location, daily turnover and number of employees, PC type (national/regional), format, availability of additional services and discount cards, type of organizational structure, efficiency of the marketing complex, implementation of the quality system, work schedule, competent management, financial condition of pharmacy, availability of an effective system of staff motivation, etc., as well as the strength of the influence of the studied factors on the level of competitiveness.

In terms of importance from the point of view of modelling the dynamics of competitiveness of small PCs (first cluster), the selected indicators were ranked. It is established that the greatest importance in modelling the dynamics of competitiveness of small PCs is taking into account such weaknesses of their activities as the lack of an effective system of employee motivation and slow response to market changes (-13.09). Less important are the round-the-clock schedule of the pharmacy (5.61) and the availability of additional services (1.74).
The factors that have the greatest positive impact on increasing the competitiveness of medium-sized PCs are highlighted: the efficiency of the marketing complex (23.72), competent management (9.22), stable financial condition (7.30) and ease of location of pharmacy (7.24). The biggest negative effects are the following factors: slow response to market changes (-9.69), the threat of absorption by larger PCs $(-7.72)$ and staff turnover $(-5.13)$.

It is established that the greatest positive impact on increasing the competitiveness of large and megaPCs have: the presence of an effective marketing complex (36.30), the presence of discount cards (35.10), the use of modern automated programs (26.49). It should be noted that in this cluster according to the results of the study were not found statistically significant negative factors.

The algorithm for developing a decision tree to select the optimal strategy to increase competitiveness for the first cluster PC (small PCs) is shown in Fig. 1.

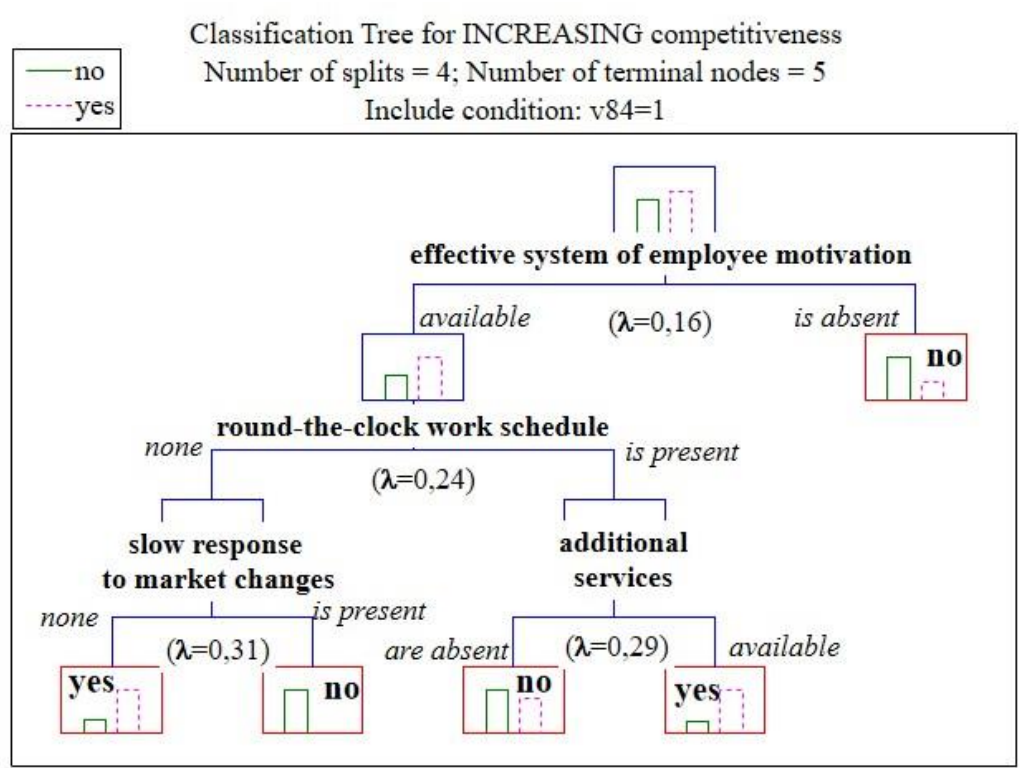

Fig. 1. Algorithm for build developing a "decision tree" to select the optimal strategy to increase competitiveness for the first cluster PC (small PCs) 
In Fig. 1 of algorithm (1) next to each predictor its weight $(\lambda)$ in the constructed model is given. From the given algorithm, it is possible to draw a conclusion that for the PC, which have entered the first cluster, increase of competitiveness can be reached, first of all, thanks to effective system of motivation of workers. This is a necessary, however, not sufficient condition for increasing competitiveness. Sufficient conditions for increasing competitiveness for small PCs are the availability of round-the-clock work schedule and additional services, or the elimination of such a weakness as the slow response of the PC to changes in the market situation. The overall accuracy of the constructed algorithm (model) for selecting the optimal strategy to increase competitiveness for small PCs is $69.5 \%$.

The corresponding algorithm of the "decision tree", built for a medium-sized PC (second cluster), is shown in Fig. 2.

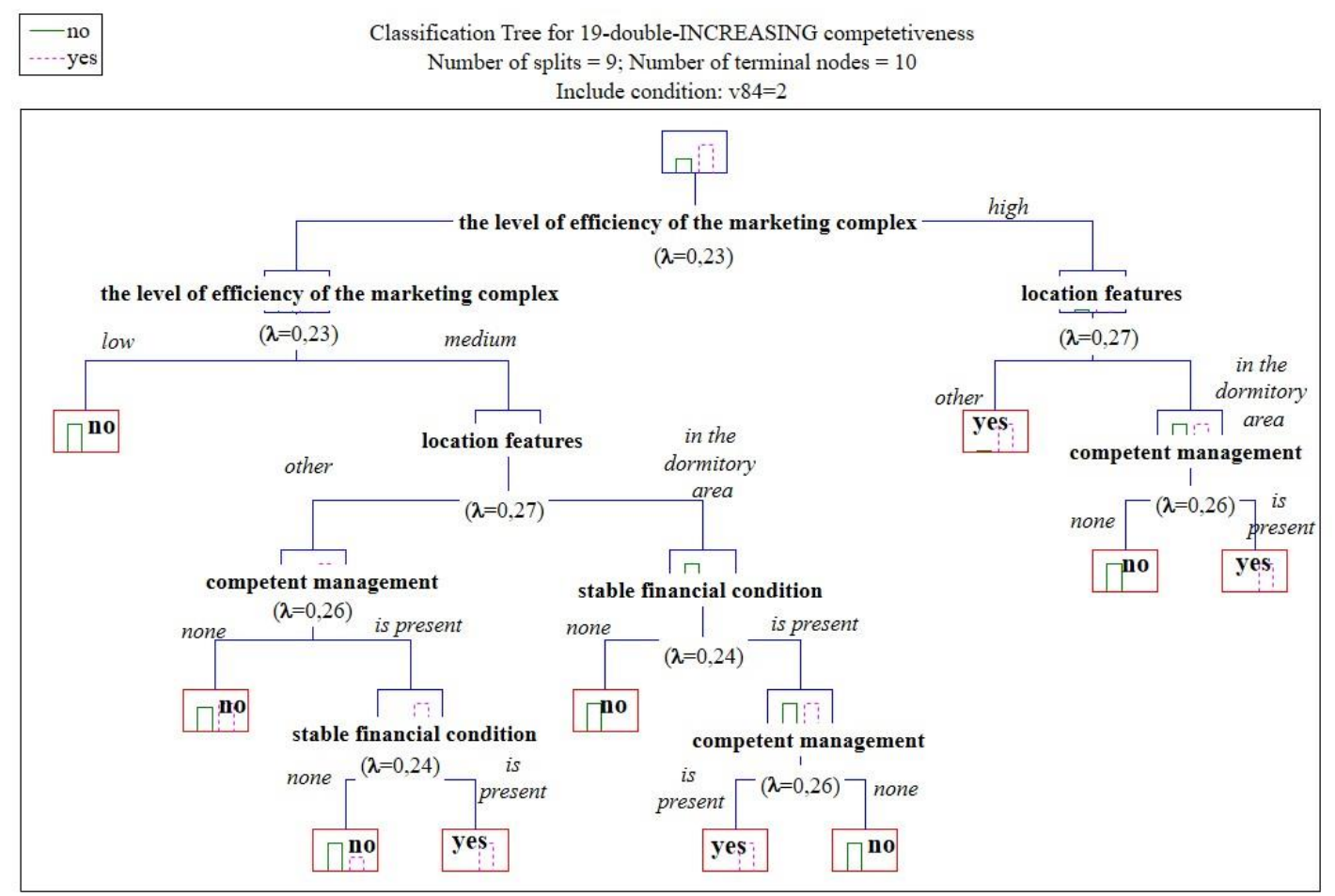

Fig. 2. Algorithm for constructing a "decision tree" to select the optimal strategy to increase competitiveness for the second cluster PC (medium PC)

As can be seen from the above algorithm, the low efficiency of the marketing complex negatively affects the level of competitiveness of PCs that form the second cluster (middle PCs). With the high efficiency of the marketing complex in most medium-sized PCs there is an increase in the level of competitiveness, but if the pharmacies of these networks are located in residential areas, a necessary condition for increasing their competitiveness is also the presence of competent PC management. If the efficiency of the marketing complex is at an average level, increasing the competitiveness of average PCs is ensured through sound management and stable financial condition. The accuracy of the constructed algorithm (model) for selecting the optimal strategy to increase the competitiveness of a medium-sized PC is $85.07 \%$.

To substantiate the optimal strategy to increase the competitiveness of large and mega-PC (third cluster), we have developed an algorithm for developing a decision tree, which is presented in Fig. 3.

The above algorithm shows that for the small share of PCs from the third cluster that do not use the system of discount cards, the formation of the optimal strategy to increase competitiveness is influenced by an effective system of employee motivation and additional services, or, in their absence, convenient location of pharmacies (next to a transport stop or exit from the subway).

Studies have shown that most large and mega-PCs use discount cards in their work. For them, the main factor in increasing competitiveness is the high level of efficiency of the marketing complex, but to ensure competitiveness in the PC should be the proper use of logistics tools, or a system of additional services. If the level of efficiency of the marketing complex in a large PC that uses discount cards is medium or low, increasing its competitiveness can be achieved through the use of modern programs for automation of basic business processes.

The accuracy of the constructed algorithm (model) for selecting the optimal strategy to increase competitiveness for the third cluster PC is $77.5 \%$.

To summarize the obtained results, the predictors used in the three models described above were used in the development of a generalized model for selecting a PC competitiveness strategy. Additionally, the PC size is included in this model as an explanatory variable. This allowed to obtain an algorithm (Fig. 4), which has a higher overall accuracy (77.55\%), specificity (77.9\%) and sensitivity $(77.2 \%)$ of determining the competitiveness of the PC, although it takes into account in less detail the factors affecting this figure for PCs of different sizes. 


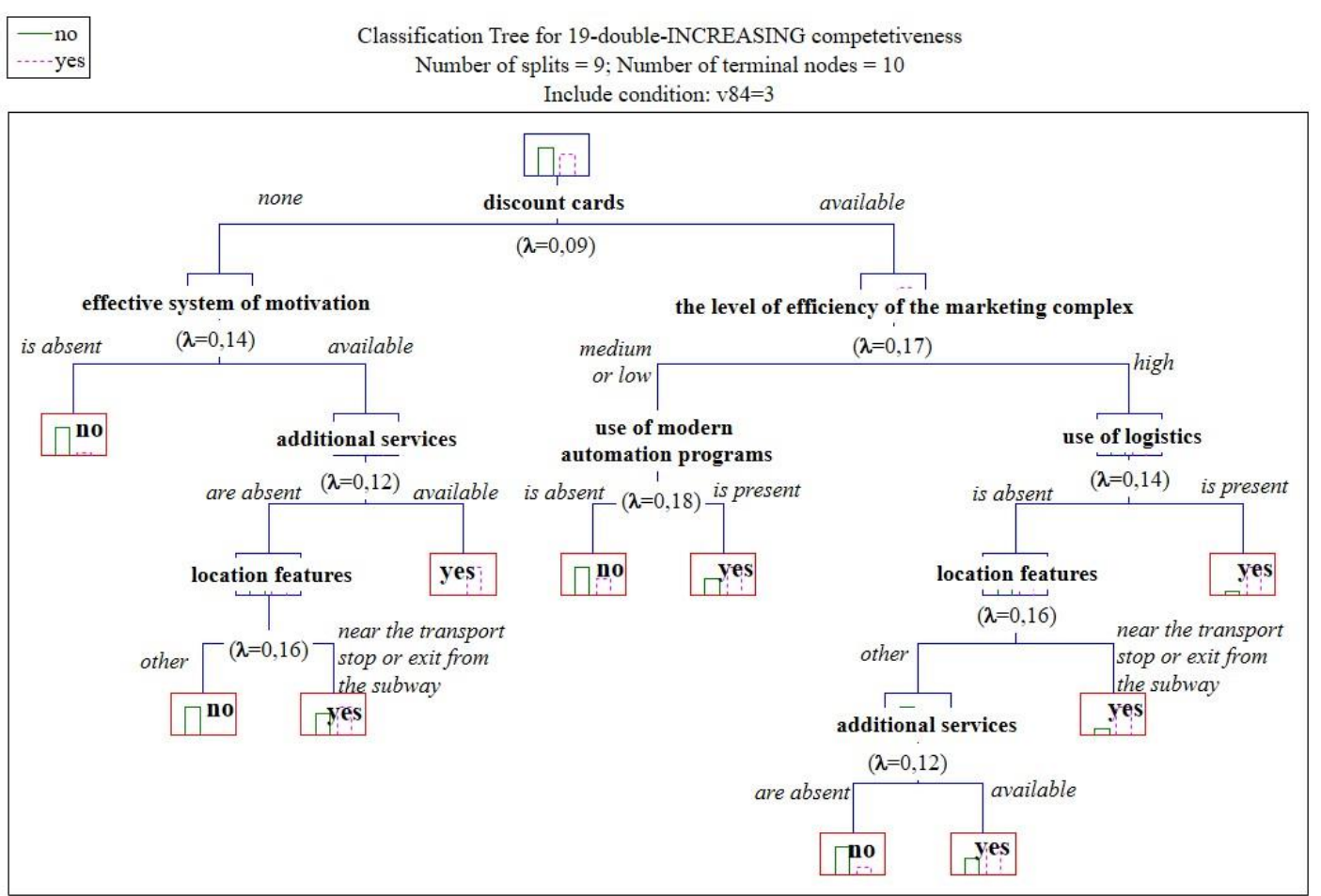

Fig. 3. Algorithm for developing a "decision tree" to select the optimal strategy to increase competitiveness for large and mega-PC (third cluster)

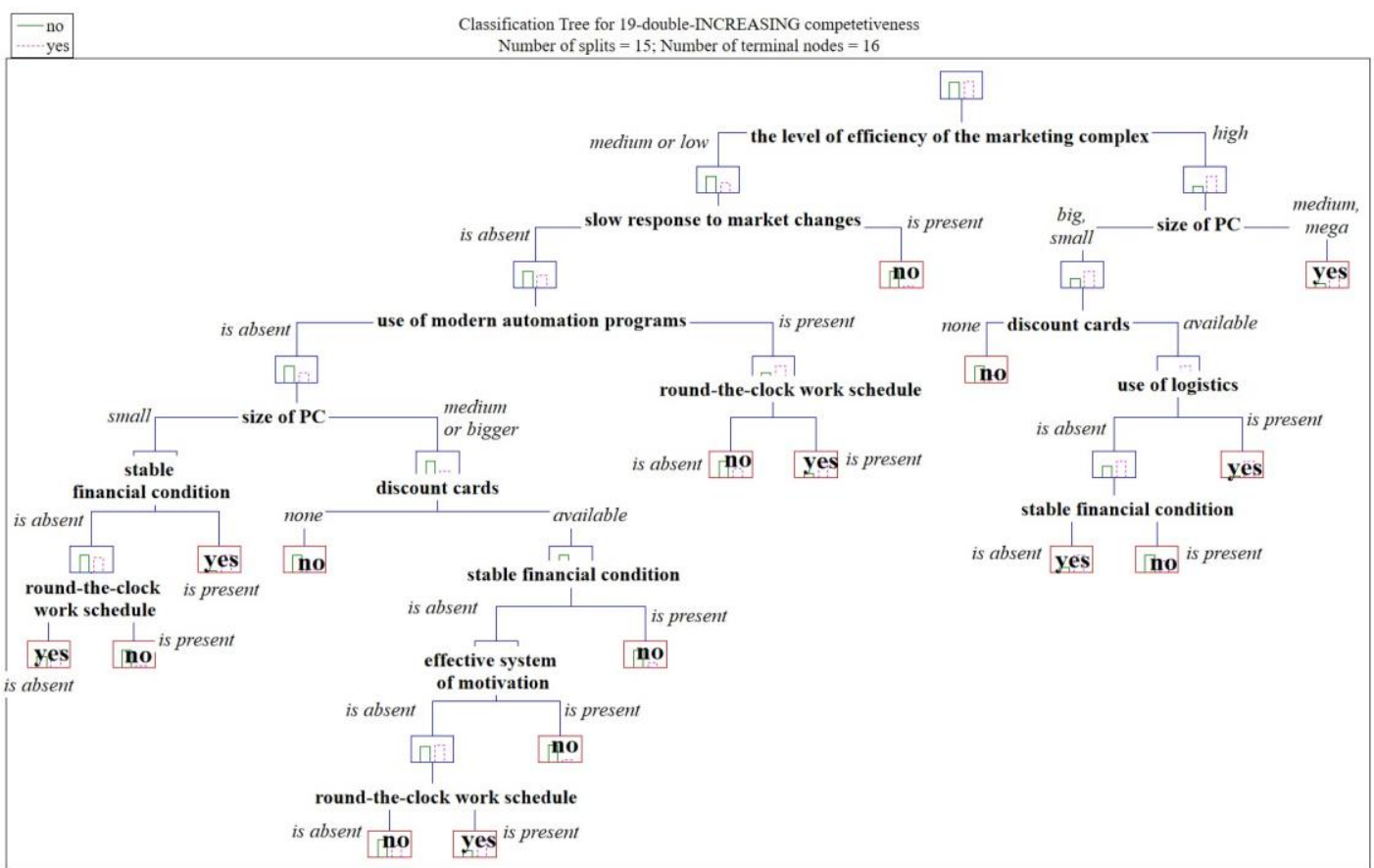

Fig. 4 Algorithm of the generalized model of "decision tree" for a choice of optimum strategy of increase of competitiveness depending on the size of the PC

According to the obtained generalized model for medium and large PCs, a high level of efficiency of the marketing complex is a sufficient condition for increasing competitiveness. With the high efficiency of the marketing complex for small and large PCs in the absence of a stable financial position to increase competitiveness, it is necessary to recommend the use of a system of discount cards, or the use of logistics.

In the absence of high efficiency of the marketing complex, a necessary condition for increasing the competi- tiveness of the PC is a timely response to market changes. In this case, regardless of the size of the PC, increasing competitiveness could be provided by a round-the-clock work schedule and the use of modern automation programs. If in these conditions small PCs do not use modern software to automate their own activities, then to ensure their competitiveness can either a stable financial position or a round-theclock work schedule. Medium and mega-PCs that do not use modern automation software can increase competitiveness with a system of discount cards, stable financial condi- 
tion, the availability of an effective system of staff motivation and round-the-clock work schedule.

\section{Discussion of research results}

It is determined that to increase the validity of management decisions aimed at increasing the competitiveness of PC in a changing market situation, it is advisable to use modelling methods based on the use of modern mathematical apparatus. To model the dynamics of PC competitiveness, depending on their size, three clusters were identified based on the values of 13 input variables - scores of the strengths of network competitiveness. It is established that all pharmacies functioning as a part of small PC form the first cluster; the second cluster - form a medium-sized PC; the third cluster - form large and mega-PC.

The indicators according to which a significant difference between clusters is established are highlighted. It was determined that in the second and third clusters (medium, large and mega-PC) experts praised such strengths of competitiveness as the availability of additional services and the convenience of the pharmacy schedule. In the first and second clusters, experts equally assessed the importance of the impact on the level of PC competitiveness of the width of the range of drugs and para-pharmaceuticals, the availability of discounts, speed and quality of service, microclimate in the team, staff training programs, staff turnover and modern automated programs. However, studies have shown that estimates of these indicators in small and medium PCs are much lower than in large and mega-PCs. This may be because small and medium PCs do not pay the necessary attention to these indicators and do not have sufficient financial resources, for example, to maintain the required breadth of the range of drugs and para-pharmaceuticals, the introduction of modern automated programs and more. Small PCs are more important than average, such factors of competitiveness as the convenience of the location of the pharmacy and the availability of prices for goods and services, the availability of a system of discounts.

In $[19,20]$ there are presented a generalized network model of supply chain oligopoly for pharmaceuticals by differentiation and network of pharmaceutical supply chains with outsourcing by price and quality competition, however, they do not consider approaches to choosing the optimal strategy to increase the competitiveness of PC depending on.

To select the optimal strategy to increase the competitiveness of PCs of different clusters, we used the method of developing a "decision tree". It is established that the biggest negative impact on the strategy of increasing the competitiveness of small PCs is a slow response to changes in market conditions; the biggest positive impact is the availability of additional services in the PC. The overall accuracy of the constructed algorithm (model) for selecting the optimal strategy to increase competitiveness for small PCs is $69.5 \%$, with an increase in competitiveness projected at exactly $66.04 \%$, no increase - at exactly $73.81 \%$.

An algorithm for selecting the optimal strategy for increasing competitiveness for a medium-sized PC (second cluster) is constructed. In the course of the study, we found that the most important factors influencing the choice of the optimal strategy to increase competitiveness for medium-sized PCs are the location of the pharmacy and competent management. Slightly less important are the presence of a stable financial condition of the PC and the level of efficiency of the marketing complex. The accuracy of the constructed algorithm (model) for choosing the strategy of increasing competitiveness for a medium-sized PC is $97.3 \%$ when determining cases of increasing competitiveness, $70 \%$ - when determining the absence of changes or declining competitiveness (overall accuracy is $85.07 \%$ ).

An algorithm for selecting the optimal strategy to increase the competitiveness of large and mega-PCs is proposed. The importance of predictor variables in the selection of the optimal strategy to increase the competitiveness of PC in the third cluster is assessed. It is established that the greatest importance for large PCs in terms of choosing the optimal strategy to increase competitiveness are the use of modern automation programs, the level of efficiency of the marketing complex and location features. Such strengths as having an effective system of employee motivation, use of logistics and availability of additional services have moderate weight in the built algorithm. The least important is the presence of discount cards. The accuracy of the constructed algorithm (model) for choosing a strategy to increase competitiveness for large and mega-PC is $72.64 \%$ when predicting an increase in competitiveness, $81.34 \%$ - when predicting its decline or invariance (overall accuracy $77.5 \%$ ).

For the generalized model of choosing the optimal strategy to increase the competitiveness of the PC, the following factors are of the greatest importance: the use of a system of discount cards $(\lambda=0.21)$ and the size of the PC $(\lambda=0.17)$; less - the use of modern automation programs $(\lambda=0.14)$ and logistics methods $(\lambda=0.11)$, the same moderate weight - round-the-clock work schedule and a high assessment of the level of efficiency of the marketing complex $(\lambda=0.097)$, the lowest - the speed of response to change market $(\lambda=0.092)$ and financial stability $(\lambda=0.084)$. The overall accuracy of the developed model is $77.55 \%$, the accuracy of forecasting the increase in competitiveness - $77.2 \%$, the absence or negative changes in competitiveness $-77.9 \%$.

Study limitations include the lack of understanding of the role and importance of some PC managers in the organization of a sound development strategy for the formation of competitive advantages and ensuring sustainable competitiveness.

Prospects for further research include the need for continuous monitoring of the impact of unstable environmental factors to form an optimal strategy to increase the competitiveness of PC. In the future, it will be useful to develop a methodology for forecasting the competitiveness of PCs, provided the choice of alternative strategies, and assess the risks associated with them, which will contribute to more informed management decisions.

\section{Conclusions}

1. To model the dynamics of PC competitiveness, depending on their size, three clusters were identified based on the values of 13 assessments of the strengths of PC competitiveness. 
2. Algorithms for selecting the optimal strategy to increase competitiveness for different sized PCs are built. It is established that the most important factors for the strategy of increasing competitiveness for small PCs are the slow response to market changes and the availability of additional services in the PC; for medium PCs - features of the pharmacy location and competent management; for large PCs - the use of modern automation programs, the level of efficiency of the marketing complex and location features.

3. The algorithm of the generalized model of "tree of decisions" for a choice of optimum strategy of increase of competitiveness depending on the size of the PC is constructed. It was found that the following factors are the most important: the size of the PC, the use of a system of discount cards, the use of modern automation programs and logistics methods, and the least - the speed of responding to market changes and financial stability. The overall accuracy of the developed model is $77.55 \%$.

4. Thus, the generalized mathematical model of the "decision tree" allows you to choose the optimal strategy to increase competitiveness for small, medium, large and mega-PC. The assessment of the importance of predictor variables allows determining the key factors in the implementation of measures aimed at implementing the chosen strategy to increase the competitiveness of PCs of different sizes.

\section{Conflict of interests}

The authors declare there is no conflict of interests.

\section{Financing}

The study was performed without financial support.

\section{References}

1. Mala, Z. V., Posylkina, O. V., Nessonova, M. M. (2017). Methodological approaches to the analysis and assessment of marketing competitive advantages of pharmacy networks. Social Pharmacy in Health Care, 3 (1), 41-51. doi: http://doi.org/10.24959/sphhcj.17.67

2. Medina, L. A., Kremer, G. E. O., Wysk, R. A. (2013). Supporting medical device development: a standard product design process model. Journal of Engineering Design, 24 (2), 83-119. doi: http://doi.org/10.1080/09544828.2012.676635

3. Ushakova, I. A., Dorokhova, L. P., Malyi, V. V., Dorokhov, A. V. (2020). Assessment of a pharmacy as a pharmaceutical service environment. Azerbaijan Pharmaceutical and Pharmacotherapy Journal, 20 (1), 24-30.

4. Niziolek, L., Chiam, T. C., Yih, Y. (2012). A simulation-based study of distribution strategies for pharmaceutical supply chains. IIE Transactions on Healthcare Systems Engineering, 2 (3), 181-189. doi: http://doi.org/10.1080/19488300.2012.709583

5. Settanni, E., Harrington, T. S., Srai, J. S. (2017). Pharmaceutical supply chain models: A synthesis from a systems view of operations research. Operations Research Perspectives, 4, 74-95. doi: http://doi.org/10.1016/j.orp.2017.05.002

6. Harrington, T. S., Phillips, M. A., Srai, J. S. (2016). Reconfiguring global pharmaceutical value networks through targeted technology interventions. International Journal of Production Research, 55 (5), 1471-1487. doi: http://doi.org/10.1080/00207543.2016.1221541

7. Mehralian, G., Zarenezhad, F., Rajabzadeh Ghatari, A. (2015). Developing a model for an agile supply chain in pharmaceutical industry. International Journal of Pharmaceutical and Healthcare Marketing, 9 (1), 74-91. doi: http://doi.org/10.1108/ijphm-09-2013-0050

8. Will M. Bertrand, J., Fransoo, J. C. (2002). Operations management research methodologies using quantitative modeling. International Journal of Operations \& Production Management, 22 (2), 241-264. doi: http://doi.org/10.1108/01443570210414338

9. Brandenburg, M., Govindan, K., Sarkis, J., Seuring, S. (2014). Quantitative models for sustainable supply chain management: Developments and directions. European Journal of Operational Research, 233 (2), 299-312. doi: http://doi.org/10.1016/j.ejor.2013.09.032

10. Narayana, S. A., Kumar Pati, R., Vrat, P. (2014). Managerial research on the pharmaceutical supply chain - A critical review and some insights for future directions. Journal of Purchasing and Supply Management, 20 (1), 18-40. doi: http://doi.org/10.1016/ j.pursup.2013.09.001

11. Chung, S. H., Kwon, C. (2016). Integrated supply chain management for perishable products: Dynamics and oligopolistic competition perspectives with application to pharmaceuticals. International Journal of Production Economics, 179, 117-129. doi: http://doi.org/10.1016/j.ijpe.2016.05.021

12. Posilkina, O., Bondarieva, I., Malyi, V., Timanyuk, I., Mala, Z. (2021). Peculiarities of effective management of products assortment depending on different sizes of pharmacy chains. ScienceRise: Pharmaceutical Science, 2 (30), 55-63. doi: http://doi.org/10.15587/ 2519-4852.2021.230287

13. Ahmadiani, S., Nikfar, S. (2016). Challenges of access to medicine and the responsibility of pharmaceutical companies: a legal perspective. DARU Journal of Pharmaceutical Sciences, 24, 124-130. doi: http://doi.org/10.1186/s40199-016-0151-z

14. Ranyard, J. C., Fildes, R., Hu, T.-I. (2015). Reassessing the scope of OR practice: The Influences of Problem Structuring Methods and the Analytics Movement. European Journal of Operational Research, 245 (1), 1-13. doi: http://doi.org/10.1016/j.ejor.2015.01.058

15. Saghiri, S., Wilding, R., Mena, C., Bourlakis, M. (2017). Toward a three-dimensional framework for omni-channel. Journal of Business Research, 77, 53-67. doi: http://doi.org/10.1016/j.jbusres.2017.03.025

16. Liu, J., Gong, Y. (Yale), Zhu, J., Zhang, J. (2018). A DEA-based approach for competitive environment analysis in global operations strategies. International Journal of Production Economics, 203, 110-123. doi: http://doi.org/10.1016/j.ijpe.2018.05.029

17. Zaki, M. J., Wagner, M. Jr. (2020). Data Mining and Machine Learning: Fundamental Concepts and Algorithms. Cambridge University Press. Available at: https://dataminingbook.info/book_html/

18. Lyman, R. O., Longnecker, M. (2010). An Introduction to Statistical Methods and Data Analysis. Brooks/Cole: Cengage Learning, 1296.

19. Masoumi, A. H., Yu, M., Nagurney, A. (2012). A supply chain generalized network oligopoly model for pharmaceuticals under brand differentiation and perishability. Transportation Research Part E: Logistics and Transportation Review, 48 (4), $762-780$. doi: http://doi.org/10.1016/j.tre.2012.01.001 
20. Nagurney, A., Nagurney, Li, L. S. (2013). Pharmaceutical supply chain networks with outsourcing under price and quality competition. International Transactions in Operational Research, 20 (6), 859-888. doi: http://doi.org/10.1111/itor.12031

Received date 28.06.2021

Accepted date 26.08.2021

Published date 31.08.2021

Iryna Bondarieva*, PhD, Associate Professor, Department of Pharmaceutical Management and Marketing, National University of Pharmacy, Pushkinska str., 53, Kharkiv, Ukraine, 61002

Volodymyr Malyi, Doctor of Pharmaceutical Sciences, Professor, Department of Pharmaceutical Management and Marketing, National University of Pharmacy, Pushkinska str., 53, Kharkiv, Ukraine, 61002

Olga Posilkina, Doctor of Pharmaceutical Sciences, Professor, PhD, Department of Management, Economics and Quality Assurance in Pharmacy, National University of Pharmacy

Pushkinska str., 53, Kharkiv, Ukraine, 61002

Zhanna Mala, PhD, Department of Pharmaceutical Management and Marketing, National University of Pharmacy, Pushkinska str., 53, Kharkiv, Ukraine, 61002

Maryna Nessonova, PhD, Associate Professor, Department of Educational and Information Technology, National University of Pharmacy, Pushkinska str., 53, Kharkiv, Ukraine, 61002

*Corresponding author: Iryna Bondarieva, e-mail: fmm@nuph.edu.ua 